doi:10.13108/2021-13-1-98

\title{
BOUNDEDNESS OF DISCRETE HILBERT TRANSFORM ON DISCRETE MORREY SPACES
}

\author{
R.A. AliEV, A.N. AHMADOVA
}

\begin{abstract}
The Hilbert transform plays an important role in the theory and practice of signal processing operations in continuous system theory because of its relevance to such problems as envelope detection and demodulation, as well as because of its use in relating the real and imaginary components, and the magnitude and phase components of spectra. The Hilbert transform is a multiplier operator and is widely used in the theory of Fourier transforms. The Hilbert transform was the motivation for the development of modern harmonic analysis. Its discrete version is also widely used in many areas of science and technology and plays an important role in digital signal processing. The essential motivation behind thinking about discrete transforms is that experimental data are most frequently not taken in a continuous manner but sampled at discrete time values. Since much of the data collected in both the physical sciences and engineering are discrete, the discrete Hilbert transform is a rather useful tool in these areas for the general analysis of this type of data.

The Hilbert transform has been well studied on classical function spaces Lebesgue, Morrey, etc. But its discrete version, which also has numerous applications, has not been fully studied in discrete analogues of these spaces. In this paper we discuss the discrete Hilbert transform on discrete Morrey spaces. In particular, we obtain its boundedness on the discrete Morrey spaces using boundedness of the Hilbert transform on Morrey spaces.
\end{abstract}

Keywords: discrete Hilbert transform, Morrey spaces, discrete Morrey spaces, boundedness.

Mathematics Subject Classification: 44A15; 40A05; 46B45; 42B35

\section{INTRODUCTION}

We denote by $l_{p}, p \geqslant 1$, the class of scalar sequences $b=\left\{b_{n}\right\}_{n \in \mathbb{Z}}$ satisfying the condition

$$
\|b\|_{l_{p}}=\left(\sum_{n \in \mathbb{Z}}\left|b_{n}\right|^{p}\right)^{\frac{1}{p}}<\infty,
$$

where $\mathbb{Z}$ is the set of integers.

Let $b=\left\{b_{n}\right\}_{n \in \mathbb{Z}} \in l_{p}, p \geqslant 1$. The sequence $\tilde{b}=\left\{\tilde{b}_{n}\right\}_{n \in \mathbb{Z}}$, where

$$
\tilde{b}_{n}=\sum_{m \neq n} \frac{b_{m}}{n-m}, \quad n \in \mathbb{Z},
$$

is called the Hilbert transform of the sequence $b=\left\{b_{n}\right\}_{n \in \mathbb{Z}}$.

M. Riesz proved (see [16]) that if $b \in l_{p}, p>1$, then $\tilde{b} \in l_{p}$ and the inequality

$$
\|\tilde{b}\|_{l_{p}} \leqslant C_{p}\|b\|_{l_{p}}
$$

R.A. Aliev, A.N. Ahmadova, Boundedness of discrete Hilbert transform on discrete Morrey SPACES.

(C) R.A. Aliev, A.N. Ahmadova. 2021.

Submitted February 14, 2020. 
holds, where $C_{p}$ is an absolute constant. Weighted analogues of 1.1 were studied investigated in works $4,6,9,12,13,15,20]$.

If $b \in l_{1}$, then the sequence $\tilde{b}$ belongs to the class $\bigcap_{p>1} l_{p}$, but generally speaking, it does not belong to the class $l_{1}$ (see [3]). In this case, R. Hunt, B. Muckenhoupt and R. Wheeden (see [12]) proved that the distribution function

$$
\tilde{b}(\lambda):=\sum_{\left\{n \in \mathbb{Z}:\left|\tilde{b}_{n}\right|>\lambda\right\}} 1
$$

of the Hilbert transform of the sequence $b$ satisfies the weak condition

$$
|\tilde{b}(\lambda)| \leqslant \frac{C_{0}}{\lambda}\|b\|_{l_{1}} \quad \text { for all } \quad \lambda>0,
$$

where $C_{0}$ is an absolute constant. In [3], it was proved that, if the sequence $b \in l_{1}$ satisfies the condition

$$
\sum_{n \in \mathbb{Z}} b_{n}=0
$$

which is necessary for the summability of the discrete Hilbert transform, and

$$
\sum_{n \in \mathbb{Z}}\left|b_{n}\right| \ln (e+|n|)<\infty
$$

then $\tilde{b} \in l_{1}$ and the following inequality holds:

$$
\|\tilde{b}\|_{l_{1}} \leqslant 6 \sum_{n \in \mathbb{Z}}\left|b_{n}\right| \ln (e+|n|)
$$

In [2], the concept of $Q$-summability of series was introduced and by using this notion, it was proved that the Hilbert transform of a sequence $b \in l_{1}$ is $Q$-summable and its $Q$-sum is equal to zero.

In this paper, we study the boundedness of the discrete Hilbert transform in the discrete Morrey spaces.

\section{Discrete Morrey SPACES}

The classical Morrey spaces $M_{\lambda, p}, 0 \leqslant \lambda \leqslant \frac{d}{p}, 1 \leqslant p<\infty$ (see $\left.1,7,14,17,19\right]$ ), consist of the functions $f \in L_{p, l o c}\left(\mathbb{R}^{d}\right)$, for which the following norm is finite

$$
\|f\|_{M_{\lambda, p}}=\sup _{x} \sup _{r>0}\left(r^{-\lambda}\|f\|_{L_{p}(B(x ; r))}\right) .
$$

We note that if $\lambda=0$, then $M_{\lambda, p}=L_{p}$; if $\lambda=\frac{d}{p}$, then $M_{\lambda, p}=L_{\infty}$ (see [1]). In the case $p>1$, $0 \leqslant \lambda<\frac{d}{p}$, F. Chiarenza and M. Frasca [8] showed the boundedness of the Hardy-Littlewood maximal operator, a fractional integral operator and a singular integral operator in the Morrey spaces. In particular, this implies the boundedness of the Hilbert transform in the Morrey spaces. It means that, in case $p>1,0 \leqslant \lambda<\frac{1}{p}$, for any $f(t) \in M_{\lambda, p}$ we have

$$
(H f)(t):=\frac{1}{\pi} \int_{\mathbb{R}} \frac{f(\tau)}{t-\tau} d \tau \in M_{\lambda, p},
$$

and there exist $C_{\lambda, p}>0$ such that

$$
\|H f\|_{M_{\lambda, p}} \leqslant C_{\lambda, p}\|f\|_{M_{\lambda, p}}
$$

holds for all $f \in M_{\lambda, p}$. 
In [10], the authors introduced a discrete analogue of the Morrey spaces and studied their inclusion properties. For $m \in \mathbb{Z}$ and $n \in \mathbb{N} \cup\{0\}$ we define

$$
S_{m, n}=\{k \in \mathbb{Z}:|k-m| \leqslant n\} .
$$

Following standard conventions, we denote the cardinality of a set $S$ by $|S|$. Then we have $\left|S_{m, n}\right|=2 n+1$ for all $m \in \mathbb{Z}$ and each $n \in \mathbb{N} \cup\{0\}$. The discrete Morrey spaces $m_{\lambda, p}$, $0 \leqslant \lambda \leqslant \frac{1}{p}, 1 \leqslant p<\infty$, consist of the sequences $b=\left\{b_{n}\right\}_{n \in \mathbb{Z}}$, for which the following norm is finite

$$
\|b\|_{m_{\lambda, p}}=\sup _{m \in \mathbb{Z}} \sup _{n \in \mathbb{N} \cup\{0\}}\left(\left|S_{m, n}\right|^{-\lambda}\left(\sum_{k \in S_{m, n}}\left|b_{k}\right|^{p}\right)^{\frac{1}{p}}\right) .
$$

In [11] it was proved the boundedness of the discrete Hardy-Littlewood maximal operators and discrete Riesz potentials on discrete Morrey spaces. We observe that if

$$
b=\left\{b_{n}\right\}_{n \in \mathbb{Z}} \in m_{\lambda, p}, \quad 1 \leqslant p<\infty, \quad 0 \leqslant \lambda<\frac{1}{p},
$$

then the series $\sum_{m \neq n} \frac{b_{m}}{n-m}$ converges absolutely. Indeed, for each $n \in \mathbb{N}$ we have

$$
\begin{aligned}
\sum_{m \neq n} \frac{\left|b_{m}\right|}{|n-m|} & =\sum_{j=1}^{\infty} \sum_{2^{j-1} \leqslant|n-m|<2^{j}} \frac{\left|b_{m}\right|}{|n-m|} \\
& \leqslant \sum_{j=1}^{\infty} \frac{1}{2^{j-1}} \sum_{|n-m|<2^{j}}\left|b_{m}\right| \\
& \leqslant \sum_{j=1}^{\infty} \frac{1}{2^{j-1}}\left(\sum_{|n-m|<2^{j}}\left|b_{m}\right|^{p}\right)^{\frac{1}{p}}\left(2^{j+1}-1\right)^{1-\frac{1}{p}} \\
& \leqslant 4\|b\|_{m_{\lambda, p}} \sum_{j=1}^{\infty} 2^{(j+1)\left(\lambda-\frac{1}{p}\right)} \leqslant \frac{4\|b\|_{m_{\lambda, p}} .}{1-2^{\lambda-\frac{1}{p}}}
\end{aligned}
$$

It follows that if

$$
b=\left\{b_{n}\right\}_{n \in \mathbb{Z}} \in m_{\lambda, p}, \quad 1 \leqslant p<\infty, \quad 0 \leqslant \lambda<\frac{1}{p},
$$

then the Hilbert transform of the sequence $b$ is well-defined.

\section{Boundedness of Discrete Hilbert transform on Discrete Morrey SPACES}

We present the main result of this paper.

Theorem 3.1. Let $1<p<\infty, 0 \leqslant \lambda<\frac{1}{p}$. For any $b \in m_{\lambda, p}$ we have $\tilde{b} \in m_{\lambda, p}$, and there exists $c_{\lambda, p}>0$ such that

holds for all $b \in m_{\lambda, p}$.

$$
\|\tilde{b}\|_{m_{\lambda, p}} \leqslant c_{\lambda, p}\|b\|_{m_{\lambda, p}}
$$

We need the following auxiliary lemma.

Lemma 3.1. If $r \in(0,1)$ and $[a, a+2 r] \subseteq[-3,3]$ then

$$
\int_{a}^{a+2 r}|\ln | t||^{p} d t \leqslant 2 \int_{0}^{r}|\ln t|^{p} d t
$$


Proof. We begin with proving the inequalities

$$
\begin{aligned}
& \int_{r}^{2 r}|\ln t|^{p} d t \leqslant \int_{0}^{r}|\ln t|^{p} d t, \quad r \in(0,1), \\
& \int_{3-2 r}^{3}|\ln t|^{p} d t \leqslant 2 \int_{0}^{r}|\ln t|^{p} d t, \quad r \in(0,1) .
\end{aligned}
$$

For $r \in\left(0, \frac{1}{2}\right]$, inequality $(3.2)$ holds thanks to the decreasing of the function $|\ln t|^{p}$ on the interval $[0,1]$, while for $r \in\left(\frac{1}{2}, 1\right)$ it is implied by the following estimates:

$$
\begin{aligned}
& \int_{r}^{2 r}|\ln t|^{p} d t<\int_{\frac{1}{2}}^{2}|\ln t|^{p} d t \leqslant \int_{\frac{1}{2}}^{2}|\ln t| d t=\frac{3}{2} \ln 2-\frac{1}{2}<\frac{2}{e}, \\
& \int_{0}^{r}|\ln t|^{p} d t>\int_{0}^{1 / e}|\ln t|^{p} d t \geqslant \int_{0}^{1 / e}|\ln t| d t=\frac{2}{e} .
\end{aligned}
$$

Let us prove (3.3). We denote

$$
\Phi(x)=2 \int_{0}^{x}|\ln t|^{p} d t-\int_{3-2 x}^{3}|\ln t|^{p} d t, \quad x \in[0,1] .
$$

Since

$$
\begin{aligned}
\Phi(0) & =0 \\
\Phi(1) & =2 \int_{0}^{1}|\ln t|^{p} d t-\int_{1}^{3}|\ln t|^{p} d t \\
& >\frac{e}{2} \int_{0}^{1 / e}|\ln t|^{p} d t+\left(2-\frac{e}{2}\right) \int_{0}^{1 / 3}|\ln t|^{p} d t-\int_{1}^{e}|\ln t|^{p} d t-\int_{e}^{3}|\ln t|^{p} d t \\
& >\frac{e}{2} \int_{0}^{1 / e}|\ln t| d t+\frac{4-e}{2}\left[\int_{0}^{1 / 9}|\ln t|^{p} d t+\int_{1 / 3}^{1 / 3}|\ln t|^{p} d t\right]-\int_{1}^{e}|\ln t| d t-\int_{e}^{3}|\ln t|^{p} d t \\
& >1+\frac{4-e}{2}\left[(2 \ln 3)^{p} \cdot \frac{1}{9}+(\ln 3)^{p} \cdot \frac{2}{9}\right]-1-(3-e)(\ln 3)^{p}>0, \\
\Phi^{\prime}(x) & =2\left(|\ln x|^{p}-|\ln (3-2 x)|^{p}\right)=0 \quad \Leftrightarrow \quad x=\frac{1}{2} \quad \text { or } x=1, \\
\Phi^{\prime}(0) & =+\infty,
\end{aligned}
$$

we get that

$$
\min _{x \in[0,1]} \Phi(x)=\min \{\Phi(0), \Phi(1)\}=0
$$

This proves 3.3 . 
Now let us prove (3.1). We denote

$$
\Psi(x)=\int_{x}^{x+2 r}|\ln t|^{p} d t, \quad x \in[0,3-2 r] .
$$

It follows from $(3.2)$ and $(3.3)$ that

$$
\begin{aligned}
& \Psi(0)=\int_{0}^{2 r}|\ln t|^{p} d t \leqslant 2 \int_{0}^{r}|\ln t|^{p} d t, \\
& \Psi(3-2 r)=\int_{3-2 r}^{3}|\ln t|^{p} d t \leqslant 2 \int_{0}^{r}|\ln t|^{p} d t, \\
& \Psi^{\prime}(x)=|\ln (x+2 r)|^{p}-|\ln x|^{p}=0 \quad \Leftrightarrow \quad x=\frac{1}{r+\sqrt{r^{2}+1}}, \\
& \Psi^{\prime}(0)=-\infty .
\end{aligned}
$$

From here we get that

$$
\max _{x \in[0,3-2 r]} \Psi(x)=\max \{\Psi(0), \Psi(3-2 r)\} \leqslant 2 \int_{0}^{r}|\ln t|^{p} d t .
$$

This shows that (3.1) holds for $a \geqslant 0$.

We proceed to the case $a<0$. If $a<0, a+r \geqslant 0$, then for each $t \in[|a|, r]$ we have

$$
\frac{1}{t} \geqslant \max \left\{2 r-t, \frac{1}{2 r-t}\right\} \text {. }
$$

Therefore, in this case,

$$
\begin{aligned}
\int_{a}^{a+2 r}|\ln | t||^{p} d t-2 \int_{0}^{r}|\ln t|^{p} d t & =\int_{0}^{|a|}|\ln t|^{p} d t+\int_{0}^{2 r-|a|}|\ln t|^{p} d t-2 \int_{0}^{r}|\ln t|^{p} d t \\
& =\int_{r}^{2 r-|a|}|\ln t|^{p} d t-\int_{|a|}^{r}|\ln t|^{p} d t \\
& =\int_{|a|}^{r}|\ln (2 r-t)|^{p} d t-\int_{|a|}^{r}|\ln t|^{p} d t \leqslant 0,
\end{aligned}
$$

and hence, (3.1) holds. If $a+r<0, a+2 r \geqslant 0$, then for each $t \in[2 r-|a|, r]$ we have

$$
\frac{1}{t} \geqslant \max \left\{2 r-t, \frac{1}{2 r-t}\right\}
$$

and in this case we get:

$$
\begin{aligned}
\int_{a}^{a+2 r}|\ln | t||^{p} d t-2 \int_{0}^{r}|\ln t|^{p} d t & =\int_{0}^{|a|}|\ln t|^{p} d t+\int_{0}^{2 r-|a|}|\ln t|^{p} d t-2 \int_{0}^{r}|\ln t|^{p} d t \\
& =\int_{r}^{|a|}|\ln t|^{p} d t-\int_{2 r-|a|}^{r}|\ln t|^{p} d t
\end{aligned}
$$




$$
=\int_{2 r-|a|}^{r}|\ln (2 r-t)|^{p} d t-\int_{2 r-|a|}^{r}|\ln t|^{p} d t \leqslant 0,
$$

that is (3.1) holds. Since the function $|\ln | t||^{p}$ is even, then in the case $a+2 r<0$ inequality (3.1) follows from the already proved case $a \geqslant 0$. This completes the proof of Lemma 3.1.

Proof of Theorem 3.1. We define the function $f(x)$ to be $2 \pi b_{k}$ for $x \in\left[k-\frac{1}{4}, k+\frac{1}{4}\right], k \in \mathbb{Z}$ and 0 elsewhere. First we are going to show that $f \in M_{\lambda, p}$. Indeed, for each $x \in\left[k-\frac{1}{2}, k+\frac{1}{2}\right)$, $k \in \mathbb{Z}$, if $r \in\left(0, \frac{1}{4}\right]$, then

$$
\begin{aligned}
r^{-\lambda}\|f\|_{L_{p}(B(x ; r))} & =r^{-\lambda}\left(\int_{x-r}^{x+r}|f(t)|^{p} d t\right)^{\frac{1}{p}} \leqslant r^{-\lambda}\left(2 r\left|2 \pi b_{k}\right|^{p}\right)^{\frac{1}{p}} \\
& =2^{1+\frac{1}{p}} \pi r^{\frac{1}{p}-\lambda}\left|b_{k}\right| \leqslant 2^{1+\frac{1}{p}} \pi\|b\|_{m_{\lambda, p}}
\end{aligned}
$$

if $r \in\left(\frac{1}{4}, 1\right]$, then

$$
\begin{aligned}
r^{-\lambda}\|f\|_{L_{p}(B(x ; r))} & =r^{-\lambda}\left(\int_{k-r}^{x+r}|f(t)|^{p} d t\right)^{\frac{1}{p}} \leqslant r^{-\lambda}\left(\frac{1}{2} \sum_{j=-1}^{1}\left|2 \pi b_{k+j}\right|^{p}\right)^{\frac{1}{p}} \\
& \leqslant 4^{\lambda} 2^{1-\frac{1}{p}} \pi\left(\left|b_{k-1}\right|^{p}+\left|b_{k}\right|^{p}+\left|b_{k+1}\right|^{p}\right)^{\frac{1}{p}} \leqslant 12^{\lambda} 2^{1-\frac{1}{p}} \pi\|b\|_{m_{\lambda, p}} ;
\end{aligned}
$$

if $r \in(n, n+1], n \in \mathbb{N}$, then

$$
\begin{aligned}
r^{-\lambda}\|f\|_{L_{p}(B(x ; r))} & =r^{-\lambda}\left(\int_{c-r}^{x+r}|f(t)|^{p} d t\right)^{\frac{1}{p}} \leqslant r^{-\lambda}\left(\frac{1}{2} \sum_{j=-n-1}^{n+1}\left|2 \pi b_{k+j}\right|^{p}\right)^{\frac{1}{p}} \\
& \leqslant n^{-\lambda} 2^{1-\frac{1}{p}} \pi\left(\sum_{j \in S_{k, n+1}}\left|b_{k+j}\right|^{p}\right)^{\frac{1}{p}} \leqslant n^{-\lambda} 2^{1-\frac{1}{p}} \pi(2 n+3)^{\lambda}\|b\|_{m_{\lambda, p}} \\
& \leqslant 5^{\lambda} 2^{1-\frac{1}{p}} \pi\|b\|_{m_{\lambda, p} .}
\end{aligned}
$$

It follows from inequalities (3.4), (3.5), (3.6) that $f \in M_{\lambda, p}$ and

$$
\|f\|_{M_{\lambda, p}}=\sup _{x} \sup _{r>0}\left(r^{-\lambda}\|f\|_{L_{p}(B(x ; r))}\right) \leqslant 12^{\lambda} 2^{1+\frac{1}{p}} \pi\|b\|_{m_{\lambda, p}} .
$$

Therefore, $H f \in M_{\lambda, p}$ and there exist $d_{0}>0$ such that

$$
\|H f\|_{M_{\lambda, p}} \leqslant d_{0}\|b\|_{m_{\lambda, p}} .
$$

We define the function $F(x)$ to be $\tilde{b}_{k}$ for $x \in\left[k-\frac{1}{2}, k+\frac{1}{2}\right), k \in \mathbb{Z}$, and

$$
G(x)=(H f)(x)-F(x) .
$$


We are going to prove that $G(x) \in M_{\lambda, p}$. For each $x \in\left[n-\frac{1}{2}, n+\frac{1}{2}\right), x \neq n \pm \frac{1}{4}, n \in \mathbb{Z}$, we have

$$
\begin{aligned}
G(x) & =\frac{1}{\pi} \int_{\mathbb{R}} \frac{f(t)}{x-t} d t-\tilde{b}_{n}=\frac{1}{\pi} \sum_{m \in \mathbb{Z}} \int_{m-\frac{1}{4}}^{m+\frac{1}{4}} \frac{2 \pi b_{m}}{x-t} d t-\sum_{m \neq n} \frac{b_{m}}{n-m} \\
& =\left(\sum_{m \neq n} 2 b_{m} \int_{m-\frac{1}{4}}^{m+\frac{1}{4}} \frac{d t}{x-t}+2 b_{n} \int_{n-\frac{1}{4}}^{n+\frac{1}{4}} \frac{d t}{x-t}\right)-\sum_{m \neq n} \frac{b_{m}}{n-m} \\
& \left.=\sum_{m \neq n} 2 b_{m} \int_{m-\frac{1}{4}}^{m+\frac{1}{4}}\left(\frac{1}{x-t}-\frac{1}{n-m}\right) d t\right)+2 b_{n} \int_{n-\frac{1}{4}}^{n+\frac{1}{4}} \frac{d t}{x-t} \\
& =G_{1}(x)+G_{2}(x) .
\end{aligned}
$$

Let $m \neq n$. Since for each $x \in\left[n-\frac{1}{2}, n+\frac{1}{2}\right)$ and $t \in\left[m-\frac{1}{4}, m+\frac{1}{4}\right]$

$$
|x-n| \leqslant \frac{1}{2}, \quad|m-t| \leqslant \frac{1}{4}, \quad|x-t| \geqslant|n-m|-|x-n|-|m-t| \geqslant|n-m|-\frac{3}{4},
$$

then we get

$$
\begin{aligned}
\left|\frac{1}{x-t}-\frac{1}{n-m}\right| & =\frac{|n-x+t-m|}{|x-t| \cdot|n-m|} \leqslant \frac{\frac{1}{2}+\frac{1}{4}}{|n-m|\left(|n-m|-\frac{3}{4}\right)} \\
& =\frac{3}{|n-m| \cdot(4|n-m|-3)} \leqslant \frac{3}{|n-m|^{2}} .
\end{aligned}
$$

Therefore, for each $x \in\left[n-\frac{1}{2}, n+\frac{1}{2}\right), x \neq n \pm \frac{1}{4}$,

$$
\mid G_{1}\left(x\left|\leqslant \sum_{m \neq n} 2\right| b_{m}\left|\int_{m-\frac{1}{4}}^{m+\frac{1}{4}}\right| \frac{1}{x-t}-\frac{1}{n-m} \mid d t \leqslant \sum_{m \neq n} \frac{3\left|b_{m}\right|}{|n-m|^{2}} .\right.
$$

It follows from inequalities 3.10 and $(2.1)$ that

$$
\left|G_{1}(x)\right| \leqslant \sum_{m \neq n} \frac{3\left|b_{m}\right|}{|n-m|^{2}} \leqslant \frac{12\|b\|_{m_{\lambda, p}}}{1-2^{\lambda-\frac{1}{p}}}
$$

If $r \in(0,1)$, then by 3.11 we obtain:

$$
\begin{aligned}
r^{-\lambda}\left\|G_{1}\right\|_{L_{p}(B(x ; r))} & =r^{-\lambda}\left(\int_{x-r}^{x+r}\left|G_{1}(x)\right|^{p} d x\right)^{\frac{1}{p}} \\
& \leqslant r^{-\lambda} \frac{12\|b\|_{m_{\lambda, p}}}{1-2^{\lambda-\frac{1}{p}}} \cdot(2 r)^{\frac{1}{p}} \leqslant \frac{12 \cdot 2^{\frac{1}{p}}}{1-2^{\lambda-\frac{1}{p}}}\|b\|_{m_{\lambda, p}} .
\end{aligned}
$$

For $r \in[k-1, k), k \in \mathbb{N}, k \geqslant 2$, by (3.10) and the Hölder inequality we have:

$$
r^{-\lambda}\left\|G_{1}\right\|_{L_{p}(B(x ; r))}=r^{-\lambda}\left(\int_{x-r}^{x+r}\left|G_{1}(x)\right|^{p} d x\right)^{\frac{1}{p}} \leqslant r^{-\lambda}\left(\int_{n-k-\frac{1}{2}}^{n+k+\frac{1}{2}}\left|G_{1}(x)\right|^{p} d x\right)^{\frac{1}{p}}
$$




$$
\begin{aligned}
& =r^{-\lambda}\left(\sum_{j=n-k}^{n+k} \int_{j-\frac{1}{2}}^{j+\frac{1}{2}}\left|G_{1}(x)\right|^{p} d x\right)^{\frac{1}{p}} \leqslant r^{-\lambda}\left(\sum_{j=n-k}^{n+k}\left(\sum_{m \neq j} \frac{3\left|b_{m}\right|}{|j-m|^{2}}\right)^{p}\right)^{\frac{1}{p}} \\
& \leqslant 3 r^{-\lambda}\left(\sum_{j=n-k}^{n+k}\left(\sum_{m \neq j} \frac{\left|b_{m}\right|^{p}}{|j-m|^{2}}\right) \cdot\left(\sum_{m \neq j} \frac{1}{|j-m|^{2}}\right)^{p-1}\right)^{\frac{1}{p}} \\
& \leqslant 12 r^{-\lambda}\left(\sum_{j=n-k}^{n+k} \sum_{m \neq j} \frac{\left|b_{m}\right|^{p}}{|j-m|^{2}}\right)^{\frac{1}{p}}=12 r^{-\lambda}\left(\sum_{m \in \mathbb{Z}}\left|b_{m}\right|^{p} \sum_{|j-n| \leqslant k, j \neq m} \frac{1}{|j-m|^{2}}\right)^{\frac{1}{p}} \\
& \leqslant 12 r^{-\lambda}\left(\sum_{m=n-2 k}^{n+2 k}\left|b_{m}\right|^{p} \cdot 4+\left.\sum_{i=1}^{\infty}\right|_{2^{i} k<|m-n| \leqslant 2^{i+1} k} ^{\frac{1}{2^{2 i-3}} k}\right)^{p} \\
& \leqslant 12 r^{-\lambda}\left(4(4 k+1)^{p \lambda}\|b\|_{m_{\lambda, p}}^{p}+\sum_{i=1}^{\infty} \frac{2\left(2^{i} k\right)^{p \lambda}\|b\|_{m_{\lambda, p}}^{p}}{2^{2 i-3} k}\right)^{\frac{1}{p}} \\
& \leqslant 12(k-1)^{-\lambda} k^{\lambda}\|b\|_{m_{\lambda, p}}\left(4 \cdot 5^{p \lambda}+\frac{16}{2^{2-p \lambda}-1}\right)^{\frac{1}{p}}
\end{aligned}
$$

and hence

$$
r^{-\lambda}\left\|G_{1}\right\|_{L_{p}(B(x ; r))} \leqslant 24\|b\|_{m_{\lambda, p}}\left(20+\frac{16}{2^{2-p \lambda}-1}\right)^{\frac{1}{p}} .
$$

It follows from (3.12), 3.13) that $G_{1} \in M_{\lambda, p}$ :

$$
\left\|G_{1}\right\|_{M_{\lambda, p}}=\sup _{x} \sup _{r>0}\left[r^{-\lambda}\left\|G_{1}\right\|_{L_{p}(B(x ; r))}\right] \leqslant d_{1}\|b\|_{m_{\lambda, p}},
$$

where

$$
d_{1}:=\max \left\{\frac{12 \cdot 2^{\frac{1}{p}}}{1-2^{\lambda-\frac{1}{p}}}, 24\left(20+\frac{16}{2^{2-p \lambda}-1}\right)^{\frac{1}{p}}\right\} .
$$

Let us show that $G_{2} \in M_{\lambda, p}$.

For each $n \in \mathbb{Z}$ we partition the set

$$
\left[n-\frac{1}{2}, n+\frac{1}{2}\right) \backslash\left\{n-\frac{1}{4} ; n+\frac{1}{4}\right\}
$$

into four parts:

$$
\left[n-\frac{1}{2}, n-\frac{1}{4}\right), \quad\left(n-\frac{1}{4}, n\right], \quad\left(n, n+\frac{1}{4}\right), \quad\left(n+\frac{1}{4}, n+\frac{1}{2}\right) .
$$

If $x \in\left[n-\frac{1}{2}, n-\frac{1}{4}\right)$, then

$$
G_{2}(x)=2 b_{n} \int_{n-\frac{1}{4}}^{n+\frac{1}{4}} \frac{d t}{x-t}=2 b_{n}\left[-\ln \left(n+\frac{1}{4}-x\right)+\ln \left(n-\frac{1}{4}-x\right)\right] .
$$

For each $x \in R$ and $\delta>0$, the identity

$$
\text { v.p. } \int_{x-\delta}^{x+\delta} \frac{d t}{x-t}=\mathrm{v} \cdot \mathrm{p} \cdot \int_{-\delta}^{\delta} \frac{d u}{-u}=0
$$


holds. Therefore, if $x \in\left(n-\frac{1}{4}, n\right]$, then

$$
\begin{aligned}
G_{2}(x) & =2 b_{n} \cdot \mathrm{v} \cdot \mathrm{p} . \int_{n-\frac{1}{4}}^{n+\frac{1}{4}} \frac{d t}{x-t}=2 b_{n}\left(\mathrm{v} \cdot \mathrm{p} \cdot \int_{x-\left(x-n+\frac{1}{4}\right)}^{x+\left(x-n+\frac{1}{4}\right)} \frac{d t}{x-t}+\int_{x+\left(x-n+\frac{1}{4}\right)}^{n+\frac{1}{4}} \frac{d t}{x-t}\right) \\
& =2 b_{n} \int_{2 x-n+\frac{1}{4}}^{n+\frac{1}{4}} \frac{d t}{x-t}=2 b_{n}\left(-\ln \left(n+\frac{1}{4}-x\right)+\ln \left(x-n+\frac{1}{4}\right)\right) .
\end{aligned}
$$

If $x \in\left(n, n+\frac{1}{4}\right)$, then

$$
\begin{aligned}
G_{2}(x) & =2 b_{n} \cdot \mathrm{v} \cdot \mathrm{p} . \int_{n-\frac{1}{4}}^{n+\frac{1}{4}} \frac{d t}{x-t}=2 b_{n}\left(\mathrm{v} \cdot \mathrm{p} . \int_{n-\frac{1}{4}}^{x-\left(n+\frac{1}{4}-x\right)} \frac{d t}{x-t}+\int_{x-\left(n+\frac{1}{4}-x\right)}^{x+\left(n+\frac{1}{4}-x\right)} \frac{d t}{x-t}\right) \\
& =2 b_{n} \int_{n-\frac{1}{4}}^{2 x-n-\frac{1}{4}} \frac{d t}{x-t}=2 b_{n}\left(-\ln \left(n+\frac{1}{4}-x\right)+\ln \left(x-n+\frac{1}{4}\right)\right) .
\end{aligned}
$$

If $x \in\left(n+\frac{1}{4}, n+\frac{1}{2}\right)$, we get:

$$
G_{2}(x)=2 b_{n} \int_{n-\frac{1}{4}}^{n+\frac{1}{4}} \frac{d t}{x-t}=2 b_{n}\left(-\ln \left(x-n-\frac{1}{4}\right)+\ln \left(x-n+\frac{1}{4}\right)\right) .
$$

This shows that for each $x \in\left[n-\frac{1}{2}, n+\frac{1}{2}\right), x \neq n \pm \frac{1}{4}$, we have

$$
G_{2}(x)=2 b_{n}\left(-\ln \left|x-n-\frac{1}{4}\right|+\ln \left|x-n+\frac{1}{4}\right|\right)
$$

and, hence,

$$
\left|G_{2}(x)\right| \leqslant\left|2 b_{n}\right|\left(\ln \frac{1}{\left|x-n-\frac{1}{4}\right|}+\ln \frac{1}{\left|x-n+\frac{1}{4}\right|}\right) .
$$

Thus, for each $n \in \mathbb{Z}$,

$$
\int_{n-\frac{1}{2}}^{n+\frac{1}{2}}\left|G_{2}(x)\right|^{p} d x \leqslant d_{2}\left|b_{n}\right|^{p}
$$

where

$$
d_{2}:=2^{p} \int_{n-\frac{1}{2}}^{n+\frac{1}{2}}\left[\ln \frac{1}{\left|x-n-\frac{1}{4}\right|}+\ln \frac{1}{\left|x-n+\frac{1}{4}\right|}\right]^{p} d x=2^{p} \int_{-\frac{1}{2}}^{\frac{1}{2}}\left[\ln \frac{1}{\left|u-\frac{1}{4}\right|}+\ln \frac{1}{\left|u+\frac{1}{4}\right|}\right]^{p} d u .
$$


Let $x \in\left[n-\frac{1}{2}, n+\frac{1}{2}\right), x \neq n \pm \frac{1}{4}$. If $r \in(0,1)$, then it follows from 3.15 and from Lemma 3.1 that

$$
\begin{aligned}
& r^{-\lambda}\left\|G_{2}\right\|_{L_{p}(B(x ; r))}=r^{-\lambda}\left(\int_{x-r}^{x+r}\left|G_{2}(t)\right|^{p} d t\right)^{\frac{1}{p}} \\
& \leqslant r^{-\lambda}\left(\int_{x-r}^{x+r}\left|2 b_{n-1}\right|^{p}\left(|\ln | t-(n-1)-\frac{1}{4}||+|\ln | t-(n-1)+\frac{1}{4}||\right)^{p} d t\right)^{\frac{1}{p}} \\
& \quad+r^{-\lambda}\left(\int_{x-r}^{x+r}\left|2 b_{n}\right|^{p}\left(|\ln | t-n-\frac{1}{4}||+|\ln | t-n+\frac{1}{4}||\right)^{p} d t\right)^{\frac{1}{p}} \\
& \quad+r^{-\lambda}\left(\int_{x-r}^{x+r}\left|2 b_{n+1}\right|^{p}\left(|\ln | t-(n+1)-\frac{1}{4}||+|\ln | t-(n+1)+\frac{1}{4}||\right)^{p} d t\right)^{\frac{1}{p}} \\
& \leqslant 24\|b\|_{m_{\lambda, p}} r^{-\lambda}\left(\int_{0}^{r}|\ln t|^{p} d t\right)^{\frac{1}{p}} \leqslant d_{3}\|b\|_{m_{\lambda, p}},
\end{aligned}
$$

where

$$
d_{3}:=24 \sup _{0<r<1} r^{-\lambda}\left(\int_{0}^{r}|\ln t|^{p} d t\right)^{\frac{1}{p}}<\infty .
$$

If $r \in[k-1, k), k \in \mathbb{N}, k \geqslant 2$, then it follows from (3.16) that

$$
\begin{aligned}
r^{-\lambda}\left\|G_{2}\right\|_{L_{p}(B(x ; r))} & =r^{-\lambda}\left(\int_{x-r}^{x+r}\left|G_{2}(x)\right|^{p} d x\right)^{\frac{1}{p}} \leqslant r^{-\lambda}\left(\int_{n-k-\frac{1}{2}}^{n+k+\frac{1}{2}}\left|G_{2}(x)\right|^{p} d x\right)^{\frac{1}{p}} \\
& =r^{-\lambda}\left(\sum_{j=n-k}^{n+k} \int_{j-\frac{1}{2}}^{j+\frac{1}{2}}\left|G_{2}(x)\right|^{p} d x\right)^{\frac{1}{p}} \leqslant r^{-\lambda}\left(\sum_{j=n-k}^{n+k} d_{2}\left|b_{j}\right|^{p}\right)^{\frac{1}{p}} \\
& \leqslant(k-1)^{-\lambda} d_{2}^{\frac{1}{p}}\|b\|_{m_{\lambda, p}}(2 k+1)^{\lambda} \leqslant 5^{\lambda} d_{2}^{\frac{1}{p}}\|b\|_{m_{\lambda, p} .}
\end{aligned}
$$

By (3.17), 3.18 we find that $G_{2} \in M_{\lambda, p}$ :

$$
\left\|G_{2}\right\|_{M_{\lambda, p}}=\sup _{x} \sup _{r>0}\left[r^{-\lambda}\left\|G_{1}\right\|_{L_{p}(B(x ; r))}\right] \leqslant d_{4}\|b\|_{m_{\lambda, p}}
$$

where

$$
d_{4}=\max \left\{5^{\lambda} d_{2}^{\frac{1}{p}}, d_{3}\right\} .
$$

Hence, owing to (3.9), 3.14) and (3.19), we conclude that $G \in M_{\lambda, p}$ :

$$
\|G\|_{M_{\lambda, p}} \leqslant\left(d_{1}+d_{4}\right)\|b\|_{m_{\lambda, p}} .
$$

Since $F(x)=(H f)(x)-G(x)$, by 3.7$)$ and 3.20 we get that $F \in M_{\lambda, p}$ :

$$
\|F\|_{M_{\lambda, p}} \leqslant\left(d_{0}+d_{1}+d_{4}\right)\|b\|_{m_{\lambda, p}} .
$$


Therefore, for each $m \in \mathbb{Z}$ and $n \in \mathbb{N} \bigcup\{0\}$,

$$
\begin{aligned}
\left|S_{m, n}\right|^{-\lambda}\left(\sum_{k \in S_{m, n}}\left|\tilde{b}_{k}\right|^{p}\right)^{\frac{1}{p}} & =(2 n+1)^{-\lambda}\left(\int_{m-n-\frac{1}{2}}^{m+n+\frac{1}{2}}|F(x)|^{p} d x\right)^{\frac{1}{p}}=(2 n+1)^{-\lambda}\|F\|_{L_{p}\left(B\left(m, n+\frac{1}{2}\right)\right)} \\
& \leqslant(2 n+1)^{-\lambda}\left(n+\frac{1}{2}\right)^{\lambda} \cdot\|F\|_{M_{\lambda, p}} \leqslant 2^{-\lambda}\left(d_{0}+d_{1}+d_{4}\right)\|b\|_{m_{\lambda, p} .}
\end{aligned}
$$

It follows that $\tilde{b} \in m_{\lambda, p}$ and

$$
\|\tilde{b}\|_{m_{\lambda, p}} \leqslant 2^{-\lambda}\left(d_{0}+d_{1}+d_{4}\right) \cdot\|b\|_{m_{\lambda, p}} .
$$

This completes the proof of Theorem 3.1.

\section{ACKNOWLEDGEMENTS}

The authors are very grateful to the referee for many useful comments and suggestions that improved the original manuscript.

\section{BIBLIOGRAPHY}

1. D.R. Adams. Morrey Spaces. Birkhäuser, Basel (2015).

2. R.A. Aliev, A.F. Amrahova. Properties of the discrete Hilbert transform // Compl. Anal. Oper. Theo. 13:8, 3883-3897 (2019).

3. R.A. Aliev, A.F. Amrahova. On the summability of the discrete Hilbert transform // Ural Math. J. 4:2, 6-12 (2018).

4. K.F. Andersen. Inequalities with weights for discrete Hilbert transforms // Canad. Math. Bul. 20:1, 9-16 (1977).

5. Y. Belov, T.Y. Mengestie, K. Seip. Discrete Hilbert transforms on sparse sequences // Proc. Lond. Math. Soc. 103:1, 73-105 (2011).

6. Y. Belov, T.Y. Mengestie, K. Seip. Unitary discrete Hilbert transforms // J. Anal. Math. 112:1, 383-393 (2010).

7. B.T. Bilalov, A.A. Huseynli, S.R. El-Shabrawy. Basis properties of trigonometric systems in weighted Morrey spaces // Azer. J. Math. 9:2, 183-209 (2019).

8. F. Chiarenza, M. Frasca. Morrey spaces and Hardy-Littlewood maximal function // Rend. Mat. Appl. 7:3-4, 273-279 (1987).

9. I. Gabisoniya, A. Meskhi. Two weighted inequalities for a discrete Hilbert transform // Proc. Razm. Math. Inst. 116, 107-122 (1998).

10. H. Gunawan, D.I. Hakim, K.M. Limanta, A.A. Masta. Inclusion properties of generalized Morrey spaces // Math. Nachr. 290:2-3, 332-340 (2017).

11. H. Gunawan, C. Schwanke. The Hardy-Littlewood maximal operator on discrete Morrey spaces // Mediterr. J. Math. 16:1, id 24 (2019).

12. R. Hunt, B. Muckenhoupt, R. Wheeden. Weighted norm inequalities for the conjugate function and Hilbert transform // Trans. Amer. Math. Sos. 176:2, 227-251 (1973).

13. E. Liflyand. Weighted estimates for the discrete Hilbert transform // in "Methods of Fourier Analysis and Approximation Theory", Birkhäuser, Basel, 59-69 (2016).

14. C.B. Morrey. On the solutions of quasi-linear elliptic partial differential equations // Trans. Amer. Math. Soc. 43:1, 126-166 (1938).

15. Y. Rakotondratsimba. Two weight inequality for the discrete Hilbert transform // Soochow J. Math. 25:4, 353-373 (1999).

16. M. Riesz. Sur les fonctions conjuguées // Math. Zeit. 27:1, 218-244 (1928).

17. W. Sickel. Smoothness spaces related to Morrey spaces - a survey. I // Eurasian Math. J. 3:3, 110-149 (2012). 
18. W. Sickel. Smoothness spaces related to Morrey spaces - a survey. II // Eurasian Math. J. 4:1, 82-124 (2013).

19. W. Sickel, D.C. Yang, W. Yuan. Morrey and Campanato meet Besov, Lizorkin and Triebel. Springer, Berlin (2010).

20. V.D. Stepanov, S.Yu. Tikhonov. Two weight inequalities for the Hilbert transform of monotone functions // Dokl. Math. 83:2, 241-242 (2011).

Rashid Avazaga Aliev,

Baku State University,

Z. Khalilov str. 23,

AZ 1148, Baku, Azerbaijan

Institute of Mathematics and Mechanics, NAS of Azerbaijan,

B. Vahabzadeh str. 9,

AZ 1148, Baku, Azerbaijan

E-mail: aliyevrashid@mail.ru

Aynur Nofel Ahmadova,

Sumgait State University,

43rd block, 1,

AZ 5008, Sumgait, Azerbaijan

E-mail: dissertant .aynur@gmail.com 